\title{
Time-of-Flight 3-D Endoscopy ${ }^{\star}$
}

\author{
Jochen Penne ${ }^{1}$, Kurt Höller ${ }^{1}$, Michael Stürmer ${ }^{1}$, Thomas Schrauder ${ }^{2}$, \\ Armin Schneider ${ }^{3}$, Rainer Engelbrecht ${ }^{2}$, Hubertus Feußner ${ }^{3}$, \\ Bernhard Schmauss ${ }^{2}$, and Joachim Hornegger ${ }^{1}$
}

1 Chair of Pattern Recognition and Erlangen Graduate School in Advanced Optical Technologies (SAOT), Friedrich-Alexander-University Erlangen-Nuremberg, Germany Jochen.Penne@informatik. uni-erlangen.de

2 Chair of Microwave Engineering and High Frequency Technology, Friedrich-Alexander-University Erlangen-Nuremberg, Germany

3 Workgroup for Minimally Invasive Therapy and Intervention, Klinikum rechts der Isar, Technical University Munich

\begin{abstract}
This paper describes the first accomplishment of the Timeof-Flight (ToF) measurement principle via endoscope optics. The applicability of the approach is verified by in-vitro experiments. Off-the-shelf ToF camera sensors enable the per-pixel, on-chip, real-time, marker-less acquisition of distance information. The transfer of the emerging ToF measurement technique to endoscope optics is the basis for a new generation of ToF rigid or flexible 3-D endoscopes. No modification of the endoscope optic itself is necessary as only an enhancement of illumination unit and image sensors is necessary. The major contribution of this paper is threefold: First, the accomplishment of the ToF measurement principle via endoscope optics; second, the development and validation of a complete calibration and post-processing routine; third, accomplishment of extensive in-vitro experiments. Currently, a depth measurement precision of $0.89 \mathrm{~mm}$ at $20 \mathrm{fps}$ with $30723-\mathrm{D}$ points is achieved.
\end{abstract}

\section{Introduction}

Minimally invasive surgery (MIS) has become a promising option if not standard procedure for a great number of surgical interventions due to the minimized collateral surgical trauma and quicker recovery. Minimally invasive intervention techniques had, have and will have a significant impact on both patients and surgeons.

Today diagnosis and intervention are based on the inspection of an image sequence acquired by a monocular video camera in MIS. This image sequence provides a flat visualization of the operation area. Consequently, depth perception and navigation of surgical instruments is very difficult. The recognition and assessment of pathological structures and tissues as well as the quantification

\footnotetext{
* The authors gratefully acknowledge funding of the Erlangen Graduate School in Advanced Optical Technologies (SAOT) by the German National Science Foundation (DFG) in the framework of the excellence initiative.
} 


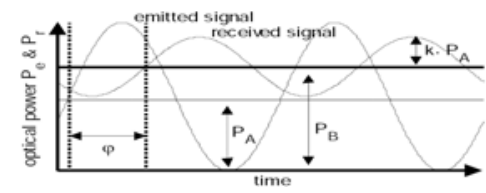

(a) ToF measurement principle

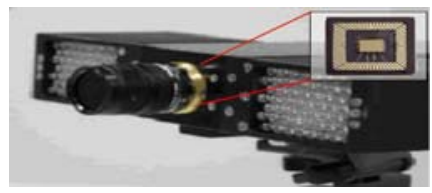

(b) ToF camera system

Fig. 1. Fig. 1(a) illustrates the ToF measurement principle. The phase-delay $\varphi$ between the sinusoidally modulated illumination light power $P_{e}$ and received light power $P_{r}$ is caused by the light propagation delay over the distance. $\varphi$ is measured in each pixel of a ToF sensor. The incoming amplitude $k \cdot P_{A}(0 \leq k \leq 1$ depending on signal attenuation) is also measured by ToF sensors and provides a grey-scale image. ( $P_{B}$ : optical power of background light sources.) Fig. 1(b) shows a commercially available ToF camera system (PMD[vision]3k-S from PMDTec $\mathrm{GmbH}$ ) and the $7.4 \mathrm{~mm} \times 6.4 \mathrm{~mm}$ ToF sensor located inside the housing. To the left and right the infrared active illumination units can be observed. Replacing the original lense by an endoscope optic and replacing the original illumination unit by a customized one were the basic hardware modification steps to build the ToF endoscope.

of their three-dimensional dimensions is only possible due to the expertise and experience of the inspecting person and is consequently subjective in its nature. By three-dimensional surface models of the operation area such tasks can be accomplished more easily, more objective and in a reproducible manner. It is a widely investigated and verified fact that 3 -D information significantly improves operative safety and precision during intervention 1 as well as during surgical training [2], for example in laparoscopic or gastrointestinal endoscopy [3].

No widely applicable and easy-to-build system is available which enables realtime, 3-D measuring (in contrast to $3 D$ visualization) at a constant pixel resolution in the operation area during an endoscopic intervention. The system proposed in this article provides this measuring capability with sub-millimeter depth precision.

\section{State of the Art}

Intra-operatively acquiring depth information is a problem which has gained significant interest especially in the field of MIS.

Image-driven monocular or stereoscopic approaches for recovering depth data of the operation area like 4/5. have been proposed. In general, these techniques unfortunately provide no real-time capability as they require the processing of an image sequence; Furthermore, they provide no guaranteed density of the computed $3 \mathrm{D}$ point cloud as the number of computed 3D points relies on the amount of detected and tracked features. Approaches have been proposed which weaken assumptions like a static field-of-view or taking care of missing data problems 677. Such image-driven approaches have been successfully applied for surgical robot-safety management or autonomous positioning of surgical instruments.

Instead of applying vision techniques the utilization of measuring techniques for directly acquiring the depth information from the operation area have been 
investigated. Utilizing a miniaturized digital holography system $512 \times 5123 \mathrm{D}$ points can be obtained at $5 \mathrm{fps}$. 8 . The authors successfully address non-medical application fields but do not comment on the applicability under sterile conditions. This is an important issue as the proposed measurement probe is mounted at the tip of the endoscope and thus has to be sterilized for intra-operative usage. In [9] and 10, an approach based on the detection of a laser beam line which is actively controlled by an optical galvano scanner is described. Approx. 4000 $3 \mathrm{D}$ points can be obtained at 5-6 fps which was verified to be enough information for a robotic navigation system. The approach requires the insertion of two monocular endoscope optics: One for the projection of the laser beam and one for observing the projected laser beam. The effort of inserting an additional endoscope optic to obtain the 3D geometry may not be suitable for practical clinical use.

Our approach aims at utilizing a single endoscope optic, which is commonly available during endoscopic interventions, to derive $3 \mathrm{D}$ information of the operation area.

A measurement principle, which can derive 3D information if only one endoscope optic is available, is the ToF measurement principle. ToF sensors have been developed without a focus on MIS or endoscopes and are available since one decade [11. ToF sensors consist of a pixel matrix and an external illumination unit, which actively illuminates the scene with an incoherent near infrared light. This light is intensity-modulated with a modulation frequency $f_{\text {mod }}$, which is usually in the range of $\leq 30 \mathrm{MHz}$ for commercially available ToF sensors. Each pixel is synchronized with the illumination unit and measures the phase-delay $\varphi$ due to the propagation delay between the emitted and reflected light. This approach is illustrated in Fig. 1(a) The phase-delay is related to the propagation time of the signal $t_{d}$ by $t_{d}=\varphi /\left(2 \pi \cdot f_{\text {mod }}\right)$, and the traveled distance $d$ of the signal can be computed by $d=c \cdot t_{d}$, where $c$ is the speed of light in the transmission medium $(c \approx 299.710 \mathrm{~km} / \mathrm{s}$ in air). Additionally, each pixel provides an amplitude information proportional to the intensity of the incoming infrared light. The amplitude information is mainly depending on the distance and the reflecting material. This information provides a gray-value image (amplitude data image) in addition to the distance map.

3D Cartesian coordinates can be computed from the measured distances. Standard calibration routines 12 can be applied to compute the necessary intrinsic camera parameters if they are not known in advance.

\section{Method and Evaluation}

\subsection{Illumination Unit of the ToF Endoscope}

For the ToF principle a light source with fast intensity modulation is required. As this modulation frequency $f_{\text {mod }}$ is in the range of $10 \mathrm{MHz}$ to $100 \mathrm{MHz}$ a mechanical modulation of the light beam by a rotating chopper wheel can not be used. Thermal light sources like tungsten incandescent lamps used typically for endoscope illumination can not be modulated that fast by the electrical 


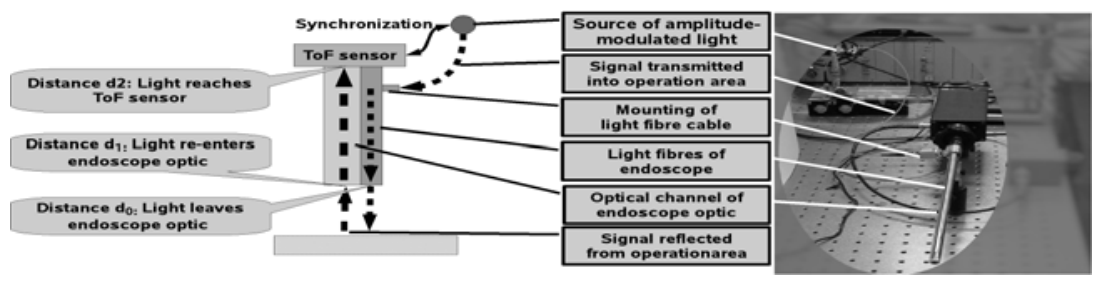

Fig. 2. Scheme of the signal transmission chain and hardware setup of a ToF endoscope. Note that $d_{0} \leq d_{1}<d_{2}$. The large dashed line depicts the transmission of the illuminating light from the light source to the operation area. The small dashed line depicts the transmission of the illuminating light from the operation area to the ToF sensor.

current, either. Therefore, light emitting diodes (LEDs) are typically applied for the illumination unit of ToF cameras as can be seen in Fig. 1(b). LEDs can be modulated by their electrical current up to $100 \mathrm{MHz}$. However, to generate a sufficient light intensity, many LEDs are required in parallel. This makes such an illumination unit impractical for coupling to the illumination fiber guide of an endoscope. For this work, a single fiber-coupled high-power laser diode has been adopted to the endoscope. With an output power of max. $2 \mathrm{~W}$ emitted from a single $200 \mu \mathrm{m}$ diameter optical fiber, this laser diode can easily be coupled to the endoscope and provides sufficient light power to overcome the transmission losses of the endoscope illumination and image guides for a good signal-to-noise ratio of the ToF camera. The high-frequency characteristics of the laser diode have been studied thoroughly to design the required high-speed driver electronics. By a single RF MOSFET transistor the modulation of the laser diode up to frequencies of $50 \mathrm{MHz}$ with potential to reach $100 \mathrm{MHz}$ for future ToF cameras with an improved distance resolution was enabled. The modulation is synchronized with the ToF camera for accurate phase measurements. Thus, a powerful and versatile illumination light source for adopting standard 3D ToF cameras to endoscopes was realized.

\subsection{Data Processing}

As Fig. 2 depicts, the distance value (if not explicitly stated otherwise all distances are specified in $\mathrm{mm}$ ) computed by a pixel of the ToF sensor is not initially the distance from the endoscope tip to the operation area, but rather biased by a constant error $d_{0}+\left(d_{2}-d_{1}\right)$.

The distance $d_{0}+\left(d_{2}-d_{1}\right)$ is different for each pixel due to slight differences in the transmission way through the fiber optics and optical channel of the endoscope optic. To remove this error an opaque object of good reflectivity (for example a sheet of white paper) is held directly before the endoscope optic: The distance measured in each pixel corresponds to $d_{0}+\left(d_{2}-d_{1}\right)=d_{2}$ as $d_{1}-d_{0}=0$. The acquired distances are additionally post-processed with a bilateral filter using a spatial sigma of 10.0 and a range sigma of 50.0 [13] to get 
a smooth 2D distance-correction mask. Subtracting the computed offset mask from the acquired distance map and dividing the obtained values by two yields the distances of the observed points from the endoscope tip. Such distances will be further on referred to as offset-corrected. To reduce outliers a $2 \mathrm{D}$ bilateral filter (with spatial sigma 1.0 and range sigma 5.0) is finally applied to the offsetcorrected distance map. Pixels whose distance measurement is severely corrupted either by acquiring very few of the infrared light or by acquiring too much of it (which leads to saturation effects in a pixel and consequently to an invalid distance measurement) can be automatically identified by applying an upper and lower threshold to the amplitude data. As the amplitude information is an absolute measurement of the acquired amplitude of the observed infrared signal a global threshold can be chosen.

\subsection{Evaluation of Measurement Precision and In-vitro Experiments}

A ToF endoscope utilizing the ToF sensor of the ToF camera PMD[vision]3kS, which operated at a modulation frequency of $30 \mathrm{MHz}$, and a zero degree endoscope optic with $10 \mathrm{~mm}$ diameter was used for the tests and experiments. The ToF sensor had a lateral resolution of $64 \times 48$ pixels and operated at upto

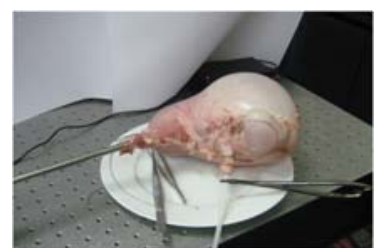

(a) In-vitro experimental setup
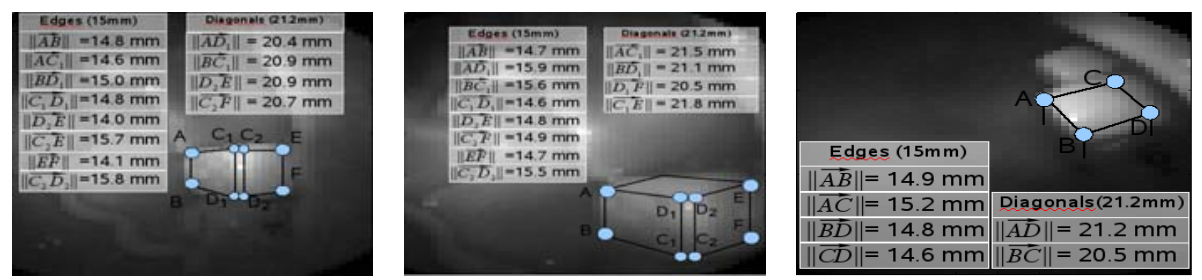

(d) 3-D measuring (Cube (e) 3-D measuring (Cube at (f) 3-D measuring (Cube at at $4.6 \mathrm{~cm}$; scene range: $3 \mathrm{~cm}$; scene range: $1.2 \mathrm{~cm}-4 \mathrm{~cm}$; scene range: $2.5 \mathrm{~cm}-$
$1.4 \mathrm{~cm}-13 \mathrm{~cm})$
$8 \mathrm{~cm})$
$10 \mathrm{~cm}$ )

Fig. 3. Fig. 3(a) shows the setup used for the experiments. Fig. 3(b) 3(c) show the data provided by the ToF endoscope: A $2 \mathrm{D}$ amplitude data image (Fig. $3(\mathrm{~b})]$ and a distance map (Fig. 3(c) the brighter a pixel the closer is the point) of a tunnel-like anatomical structure at the entrance of the stomach. Fig. 3(d) 3(f) Endoscopic 3-D measurement of edge and surface diagonal length of a $15 \times 15 \times 15 \mathrm{~mm}$ cube from different viewing positions in the porcine stomach. The depicted gray value image encodes the amplitude data (not the distances) provided by the ToF endoscope. 


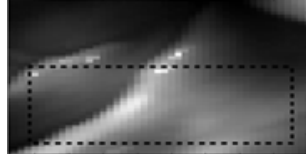

(a) Amplitude image of gastric mucosa

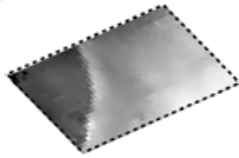

(b) Distance map of ROI

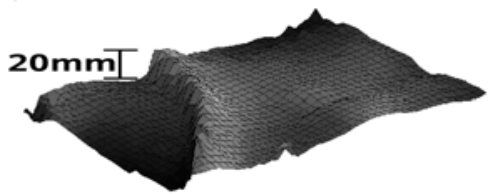

(c) 3D Visualization of ROI

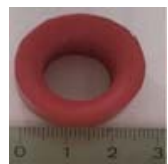

(d) Object (e) $2 \mathrm{D}$ dimension image

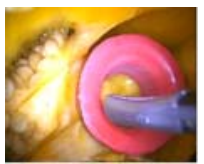

Color

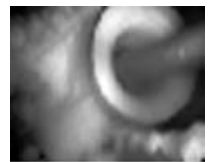

(f) ToF amplitude image

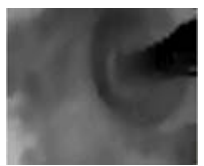

dis- (h) 3D Visual-

$\begin{array}{lr}\text { (g) } \text { ToF }^{-} & \text {dis) } 3 \mathrm{D} \\ \text { tance map } & \text { ization }\end{array}$

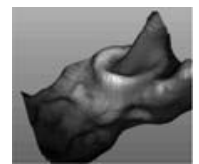

Fig. 4. Various example data sets. Porcine stomach experiments: Fig. 4(a) Amplitude data with Region-of-Interest(ROI); Fig. 4(b) Distance map of ROI; Fig 4(c) 3D visualization. 2D endoscopic color data vs. ToF endoscopic 3D data: A ring-shaped object (Fig. 4(d) was inserted into a pepper (Fig. 4(e) 2D endoscopic color image). ToF endoscope data: Amplitude data (Fig. 4(f) , depth map (Fig. 4(g)), 3D Visualization (Fig. 4(h) .

25 fps. If not explicitly stated otherwise, the data (acquired using the ToF sensor) which is referred to in the following has been acquired by a calibrated endoscope optic and the distances have been offset-corrected. For a working distance of $3 \mathrm{~cm}$ the measurement precision was computed. The measurement expression is expressed for each pixel by the standard deviation of the acquired distances when observing a static scene. An average precision of $0.89 \mathrm{~mm}$ and a median precision of $0.71 \mathrm{~mm}$ was computed from 100 acquired distance maps. A lower amplitude threshold of 50 and an upper threshold of 700 were chosen to detect pixels with a severely corrupted distance measurement (see section 3.2). $5 \%$ of the pixels were neglected. An empty porcine stomach was manually insufflated with air. The ToF endoscope was inserted via the remaining parts of the esophagus. Before inserting the endoscope optic two plastic cubes each of size $15 \times 15 \times 15 \mathrm{~mm}$ were inserted into the stomach. This served the purpose to be able to observe three-dimensionally objects of known size and shape. During the experiments one of the cubes was observed from different viewing positions. The four 3$\mathrm{D}$ boundary points of each visible surface square were manually selected. The length of the edges (ground truth: $15 \mathrm{~mm}$ ) of the square as well as the length of its diagonals (ground truth: $21.2 \mathrm{~mm}$ ) were computed. The $3-\mathrm{D}$ point coordinates of the selected points were utilized. Thus, the computed lengths are 3D Euclidean distances between the selected 3-D points. The results along with illustrative examples of the amplitude and distance data provided by a ToF endoscope are depicted in Fig. 3. A 3D surface reconstruction of the stomach mucosa is given in Fig. 4 along with a comparison of 2D endoscopic color data and ToF endoscopic $3 \mathrm{D}$ data. 


\section{Discussion and Conclusion}

The hardware costs for turning an available endoscope optic into a ToF endoscope utilizing only commercially available components are less than 5000 Euro (\$7000). The ToF endoscope operates at 20 fps including data acquisition and the complete processing chain (dual-core $2.4 \mathrm{GHz}$ PC, 2GB RAM). The mean measurement precision of $0.89 \mathrm{~mm}$ is sufficient to provide valuable intra-operative information. The immediately available distance map of the operation area enables a direct visual depth observation. The capability to three-dimensionally measure anatomical structures (tumors, etc.) in the operation area was verified by using an artificially inserted cube of known dimensions (see Fig. 3(d) 3(f)].

ToF endoscopes introduce outstanding perspectives to minimally invasive surgery. Approaches addressing collision detection, robot-guided surgery, intraoperative navigation support and 3-D visualization of the operation area will benefit from the proposed novel 3-D endoscope. Additionally, the capability to measure distances and dimensions of user-selected anatomical structures threedimensionally in real-time in the operation area has the capability to provide valuable diagnostic information to the surgeon. Innovative new endoscopic intervention techniques like NOTES (Natural Orifice Transluminal Endoscopic Surgery) may be accomplished more successfully by utilizing ToF endoscopes. Considering the ToF measurement principle and endoscope optics there are no restrictions: Any rigid or non-rigid endoscope optic can be utilized as a ToF endoscope in the proposed manner. Only chip-on-tip endoscopes can not be used in such a way.

\section{Outlook}

Currently, the utilized infrared illumination unit does not meet the safety requirements for being utilized outside a controlled laboratory. An illumination operating in the visible spectral range would provide two advantages: a better quantum efficiency of ToF sensors and the eye-safety requirements are somewhat less restrictive in the range of visible light.

Next generation ToF sensors will provide lateral resolutions of $204 \times 204$ pixels. The utilization of such sensors for a ToF endoscopes will improve the observability of smaller anatomical structures. By mounting a beam-divider and an additional standard CCD image sensor, 2D color information and distance information can be acquired with one endoscope optic. The presented experiments have been accomplished with a rigid endoscope optic. A quantitative evaluation of the capabilities of a flexible ToF endoscope is subject to current research.

\section{References}

1. Wengert, C., Bossard, L., Häberling, A., Baur, C., Székely, G., Cattin, P.C.: Endoscopic Navigation for Minimally Invasive Suturing. In: Ayache, N., Ourselin, S., Maeder, A. (eds.) MICCAI 2007, Part II. LNCS, vol. 4792, pp. 620-627. Springer, Heidelberg (2007) 
2. Votanopoulos, K., Brunicardi, F., Thornby, J., Bellows, C.: Impact of threedimensional vision in laparoscopic training. World Journal Of Surgery 32(1), 110 118 (January 2008)

3. Yoshida, T., Inoue, H., Hara, E., Umezawa, A., Ohtsuka, K., Endo, S., Tamegai, Y., Kashida, H., Tanaka, J., Kudo, S.: Newly developed 3D endoscopic system: preliminary experience. Endoscopy 35(2), 181-184 (2003)

4. Burschka, D., Li, M., Taylor, R., Hager, G.D.: Scale-Invariant Registratiou of Monocular Endoscopic Images to CT-Scans for Sinus Surgery. In: Barillot, C., Haynor, D.R., Hellier, P. (eds.) MICCAI 2004. LNCS, vol. 3217, pp. 413-421. Springer, Heidelberg (2004)

5. Mountney, P., Stoyanov, D., Davison, A.J., Yang, G.-Z.: Simultaneous Stereoscope Localization and Soft-Tissue Mapping for Minimal Invasive Surgery. In: Larsen, R., Nielsen, M., Sporring, J. (eds.) MICCAI 2006. LNCS, vol. 4190, pp. 347-354. Springer, Heidelberg (2006)

6. Hu, M., Penney, G., Edwards, P., Figl, M., Hawkes, D.: 3D Reconstruction of Internal Organ Surfaces for Minimal Invasive Surgery. In: Ayache, N., Ourselin, S., Maeder, A. (eds.) MICCAI 2007, Part I. LNCS, vol. 4791, pp. 68-77. Springer, Heidelberg (2007)

7. Lo, B., Scarzanella, M.V., Stoyanov, D., Yang, G.Z.: Belief Propagation for Depth Cue Fusion in Minimally Invasive Surgery. In: Metaxas, D., Axel, L., Fichtinger, G., Székely, G. (eds.) MICCAI 2008, Part II. LNCS, vol. 5242, pp. 104-112. Springer, Heidelberg (2008)

8. Kolenovic, E., Osten, W., Klattenhoff, R., Lai, S., von Kopylow, C., Jüptner, W.: Miniaturized Digital Holography Sensor for Distal Three-Dimensional Endoscopy. Appl. Opt. 42(25), 5167-5172 (2003)

9. Hayashibe, M., Suzuki, N., Nakamura, Y.: Laser-scan endoscope system for intraoperative geometry acquisition and surgical robot safety management. Medical Image Analysis 10(4), 509-519 (2006); Special Issue on Functional Imaging and Modelling of the Heart (FIMH 2005)

10. Hayashibe, M., Suzuki, N., Hattori, A., Nakamura, Y.: Intraoperative Fast 3D Shape Recovery of Abdominal Organs in Laparoscopy. In: Dohi, T., Kikinis, R. (eds.) MICCAI 2002. LNCS, vol. 2489, pp. 356-363. Springer, Heidelberg (2002)

11. Xu, Z., Schwarte, R., Heinol, H., Buxbaum, B., Ringbeck, T.: Smart Pixel - Photometric Mixer Device (PMD) / New System Concept of a 3D-Imaging-on-a-Chip. In: $5^{\text {th }}$ International Conference on Mechatronics and Machine Vision in Practice, Nanjing, pp. 259-264 (1998)

12. Zhang, Z.: A Flexible New Technique For Camera Calibration. IEEE Transactions on Pattern Analysis and Machine Intelligence 22(11), 1330-1334 (2000)

13. Tomasi, C., Manduchi, R.: Bilateral Filtering for Gray and Color Images. In: Sixth International Conference on Computer Vision (ICCV 1998), pp. 839-846 (1998) 